\title{
Effect of Colesevelam HCl Monotherapy on Lipid Particles in Type 2 Diabetes Mellitus
}

\author{
Robert S. Rosenson • Scott P. Rigby • Michael R. Jones • \\ Hubert S. Chou \\ Published online: 8 April 2014 \\ (C) The Author(s) 2014. This article is published with open access at Springerlink.com
}

\begin{abstract}
Purpose In addition to lowering hemoglobin A1C, colesevelam has been shown to improve the atherogenic lipoprotein profile of subjects with type 2 diabetes mellitus (T2DM) when used in combination with metformin and/or sulfonylureas. A recent study evaluated the effects of colesevelam as antidiabetes monotherapy in adults with T2DM who had inadequate glycemic control (hemoglobin A1C $\geq 7.5$ to $\leq 9.5 \%$ ) with diet and exercise alone; we report here the effects on lipoprotein particle subclasses.

Methods Subjects were randomized to receive oral colesevelam $3.75 \mathrm{~g} /$ day $(n=176)$ or placebo $(n=181)$ for 24 weeks. Changes in lipoprotein particle subclasses were determined by nuclear magnetic resonance spectroscopy.
\end{abstract}

R. S. Rosenson $(\bowtie)$

Cardiometabolic Disorders, Mt. Sinai Heart, Icahn School of

Medicine at Mount Sinai, 1425 Madison Avenue, MC1 Level,

New York, NY 10029, USA

e-mail: robert.rosenson@mssm.edu

S. P. Rigby

Summit Research Group, 4466 Darrow Road, Building B, Suite 5, Stow, OH 44224, USA

\section{S. P. Rigby}

Unity Health Network, LLC, 307 W. Main Street, Suite B, Kent, OH 44240, USA

M. R. Jones

Daiichi Sankyo, Inc., 2 Hilton Court, Parsippany, NJ 07054, USA

H. S. Chou

Daiichi Sankyo Pharma Development, 399 Thornall Street, Edison, NJ 08837, USA

Present Address:

M. R. Jones

Healthcare Consultant, 9156 Pumpkin Ridge, Port St. Lucie, FL 34986, USA
Results At Week 24 with last observation carried forward, colesevelam produced a reduction in total low-density lipoprotein (LDL) particle concentration (baseline: 1,611 nmol/L; least-squares [LS] mean treatment difference: $-143 \mathrm{nmol} / \mathrm{L}$, $p<0.0001)$ versus placebo; reductions were also seen in large, small, and very small LDL particle concentrations (all $p<0.05$ ). There was also a reduction in total very lowdensity lipoprotein (VLDL) and chylomicron particle concentration (baseline: $88 \mathrm{nmol} / \mathrm{L}$; LS mean treatment difference: $-1 \mathrm{nmol} / \mathrm{L}, p=0.82$ ) that resulted from a lowering in small VLDL particle concentration (baseline: $45 \mathrm{nmol} / \mathrm{L}$; LS mean treatment difference: $-5 \mathrm{nmol} / \mathrm{L}, p=0.03$ ). In addition, with colesevelam there was an increase in total high-density lipoprotein (HDL) particle concentration versus placebo (baseline: $31 \mu \mathrm{mol} / \mathrm{L}$; LS mean treatment difference: $+0.6 \mu \mathrm{mol} / \mathrm{L}$, $p=0.20$ ), due to increases in the large (baseline: $5 \mu \mathrm{mol} / \mathrm{L}$; LS mean treatment difference: $+0.5 \mu \mathrm{mol} / \mathrm{L}, p=0.007)$ and medium (baseline: $3 \mu \mathrm{mol} / \mathrm{L}$; LS mean treatment difference: $+0.8 \mu \mathrm{mol} / \mathrm{L}, p=0.02$ ) HDL subclasses.

Conclusions Colesevelam monotherapy in subjects with T2DM resulted in generally favorable changes in certain lipoprotein subclass profiles compared with placebo.

Keywords Colesevelam $\cdot$ Monotherapy $\cdot$ Lipoprotein particles $\cdot$ Low-density lipoprotein $\cdot$ Nuclear magnetic resonance $\cdot$ Type 2 diabetes mellitus

\section{Introduction}

Insulin resistance and type 2 diabetes mellitus (T2DM) are associated with a variety of lipid and lipoprotein abnormalities, including reduced high-density lipoprotein (HDL) cholesterol levels, elevated triglyceride levels, and an altered distribution of lipoprotein particles [1]. Patients with insulin resistance also have alterations to the lipoprotein subclass 
profile that are not reflected by standard lipid panels [2, 3]. In particular, very low-density lipoprotein (VLDL) particle concentration (VLDL-P) and low-density lipoprotein (LDL) particle concentration (LDL-P) are increased and HDL particle concentration (HDL-P) is decreased in patients with insulin resistance or T2DM compared with individuals who have normal insulin sensitivity [2]. Among the lipoprotein subclasses, VLDL particle sizes are increased and LDL and HDL particle sizes are decreased in patients with insulin resistance or T2DM. These effects are seen despite normal or near-normal levels of LDL cholesterol and total cholesterol [3].

This altered distribution of lipoprotein particles (particularly LDL particles) is relevant to cardiovascular disease (CVD) risk [4]. LDL-P is predictive for coronary endothelial dysfunction and CVD risk [5, 6] and may be a better predictor than LDL cholesterol, especially in patients with insulin resistance or T2DM [4, 7-9]. In addition, HDL-P is inversely correlated with carotid intima-media thickness and coronary heart disease risk [10-12]. Consequently, reducing total LDL-P through changes in the distribution of lipoprotein particles in patients with hypercholesterolemia via intervention with lipidlowering agents is a potentially good therapeutic strategy to reduce CVD risk [13]. However, it should be noted that although clinical data exist demonstrating the ability of some cholesterol-lowering drugs to improve the LDL subclass distribution profile, the supporting data come from predominately smaller studies. Thus, the ability of these agents to reduce vascular risk still needs to be demonstrated in large clinical trials. For example, it is important to determine the clinical benefit of drug regimens that reduce small LDL particle fractions versus those that reduce all fractions simultaneously, as well as to identify the subclass distribution profile produced by each lipid-lowering agent, so that an agent may be chosen to appropriately modify the lipid profile of the individual patient [14]. In addition, determining which agents increase HDL-P is also important, as HDL-P is inversely correlated with carotid intima-media thickness and cardiovascular events, and therefore administering an agent that increases HDL-P may help to further reduce CVD risk $[10-12,15]$.

The bile acid sequestrant colesevelam has been shown to improve glycemic and lipid parameters in subjects with T2DM when added to metformin-, sulfonylurea-, or insulinbased therapy [16-19]. In addition, treatment with colesevelam in combination with metformin and/or sulfonylureas has been shown to result in a more beneficial lipoprotein profile in subjects with T2DM [20,21]. A recent study evaluated the effects of colesevelam as an antidiabetes monotherapy in adults with T2DM who were inadequately controlled by diet and exercise alone (ClinicalTrials.gov identifier: NCT00789737) [22]. Here we report the effects of colesevelam monotherapy on the concentrations and sizes of various lipoprotein particle subclasses from this study.

\section{Materials and Methods}

\section{Study Design}

This was a 24-week, randomized, double-blind, placebocontrolled, parallel-group study conducted at approximately 80 study centers in the United States. The study was conducted in compliance with independent ethics committee/ institutional review board regulations, good clinical practice guidelines, and the principles of the Declaration of Helsinki. Institutional review board approval was obtained before initiation of the study, and all subjects provided written informed consent.

Details of this study, including design, inclusion/exclusion criteria, and statistical methods, have been described previously [22]. Briefly, the study enrolled subjects aged $\geq 18$ years with a diagnosis of T2DM (based on American Diabetes Association diagnostic criteria) who were untreated at the time of screening (i.e. treatment-naïve or not receiving antihyperglycemic medication within $\geq 3$ months prior to screening). Subjects were required to have a hemoglobin A1C (A1C) level $\geq 7.5 \%$ and $\leq 9.5 \%$ at screening, a fasting plasma glucose (FPG) level $\leq 240 \mathrm{mg} / \mathrm{dL}$ at randomization, and a fasting C-peptide level $>0.5 \mathrm{ng} / \mathrm{mL}$ at screening. Subjects were not randomized if they had persistent FPG levels $>240 \mathrm{mg} / \mathrm{dL}$ during the placebo lead-in period.

Following a 2-week single-blind placebo lead-in period, subjects were randomized to receive oral colesevelam $3.75 \mathrm{~g} /$ day $(6 \times 625 \mathrm{mg}$ tablets $)$ or placebo for 24 weeks. Subjects taking concomitant medications must have been at stable doses for $\geq 30$ days prior to enrollment and not have been anticipated to require adjustment during the study period. To the extent possible, subjects were instructed to maintain the same dosages of established lipid-lowering agents throughout the double-blind treatment period to minimize confounding the evaluation of the lipid-lowering effects of colesevelam versus placebo.

\section{Assessments}

Scheduled clinic visits were at Weeks $0,4,8,16$, and 24 after an overnight fast. Samples for chemistry, hematology, A1C, and FPG were collected at screening and every visit from baseline (Week 0) to the end of treatment (Week 24). The primary efficacy variable was A1C level at Week 24. Secondary efficacy variables included FPG, high-sensitivity C-reactive protein, total cholesterol, LDL cholesterol, HDL cholesterol, non-HDL cholesterol, triglyceride, apolipoprotein A-I (apoA-I), and apoB levels. A standard meal tolerance test was conducted at baseline and at Week 24 to evaluate the glucose excursion. Fasting blood samples were obtained for the assessment of lipid particle parameters at baseline and Week 24. Particle parameters were determined by nuclear magnetic resonance 
(NMR) spectroscopy [23]. In this technique, distributions and concentrations of individual lipid particle subclasses are determined based on the fact that lipoprotein particles within a specified diameter range emit a distinctive magnetic resonance signal with its signal intensity proportional to lipid mass concentration [14, 24]. This analysis evaluated changes in lipid particle parameters (mean lipoprotein particle concentrations and sizes) from baseline to Week 24.

\section{Statistics}

The intention-to-treat (ITT) population was the primary population for analysis, defined as all randomized subjects who received $\geq 1$ dose of study medication and had an A1C or FPG measurement at baseline and $\geq 1$ post-baseline measurement prior to taking any antihyperglycemic rescue medication. The analysis compared changes from baseline between colesevelam and placebo to rescue or Week 24 with last observation carried forward (LOCF) using the ITT population. Between-group differences were evaluated using an analysis of covariance model, with treatment as a fixed effect and baseline as a covariate; all statistical tests were 2-sided at a
$5 \%$ significance level. Because of the low number of subjects on HMG-CoA reductase inhibitors (statins), the results were not analyzed separately according to those subjects taking both a statin and colesevelam versus colesevelam without a statin.

\section{Results}

\section{Subjects}

A total of 357 subjects were randomized to receive colesevelam $(n=176)$ or placebo $(n=181)$; the ITT population comprised 344 subjects (175 and 169, respectively). Demographic and baseline characteristics of all randomized subjects were generally similar between treatment groups, as summarized in Table 1. The mean age was 52.2 years and a high percentage of subjects were Hispanic/Latino (46.5\%). The mean duration of T2DM was 4.1 years and the mean body mass index was $31.9 \mathrm{~kg} / \mathrm{m}^{2}$. Overall, $14.2 \%$ of subjects in the ITT population were taking statins and $2.9 \%$ were taking fibrates. Mean changes in weight from baseline to end of study/early termination were similar for subjects in both the
Table 1 Demographic and baseline characteristics (all randomized subjects)
$H D L$ high-density lipoprotein, $L D L$ low-density lipoprotein, $S D$ standard deviation

${ }^{a}$ There were no statistically significant between-group differences for any demographic variable or baseline variable

\begin{tabular}{|c|c|c|}
\hline Characteristic $^{\mathrm{a}}$ & Colesevelam $(n=176)$ & Placebo $(n=181)$ \\
\hline Age (years), mean (SD) & $52.6(10.25)$ & $51.8(10.52)$ \\
\hline \multicolumn{3}{|l|}{ Sex, $n(\%)$} \\
\hline Male & $94(53.4)$ & $89(49.2)$ \\
\hline \multicolumn{3}{|l|}{ Race, $n(\%)$} \\
\hline Caucasian & $122(69.3)$ & $131(72.4)$ \\
\hline Black & $27(15.3)$ & $29(16.0)$ \\
\hline Asian & $12(6.8)$ & $8(4.4)$ \\
\hline American Indian/Alaskan native & $12(6.8)$ & $11(6.1)$ \\
\hline Native Hawaiian/Pacific Islander & $1(0.6)$ & $0(0.0)$ \\
\hline Other & $2(1.1)$ & $2(1.1)$ \\
\hline \multicolumn{3}{|l|}{ Ethnicity, $n(\%)$} \\
\hline Hispanic/Latino & $86(48.9)$ & $80(44.2)$ \\
\hline Not Hispanic/Latino & $90(51.1)$ & $101(55.8)$ \\
\hline Body mass index $\left(\mathrm{kg} / \mathrm{m}^{2}\right)$, mean $(\mathrm{SD})$ & $32.0(6.50)$ & $31.8(4.94)$ \\
\hline Hemoglobin A1C (\%), mean (SD) & $8.25(0.684)$ & $8.18(0.697)$ \\
\hline Fasting plasma glucose, mg/dL, mean (SD) & $172.5(46.44)$ & $168.0(37.61)$ \\
\hline Fasting insulin, $\mu \mathrm{IU} / \mathrm{mL}$, mean (SD) & $17.7(16.63)$ & $17.9(14.04)$ \\
\hline Duration of type 2 diabetes (years), mean (SD) & $4.3(4.69)$ & $3.9(4.39)$ \\
\hline Total cholesterol (mg/dL), mean (SD) & $202.9(39.69)$ & $200.6(40.37)$ \\
\hline LDL cholesterol (mg/dL), mean (SD) & $122.5(33.80)$ & $119.0(33.17)$ \\
\hline HDL cholesterol (mg/dL), median (range) & $43.0(23.0-84.5)$ & $43.5(24.0-100.0)$ \\
\hline Non-HDL cholesterol (mg/dL), mean (SD) & $159.4(38.39)$ & $155.8(39.83)$ \\
\hline Triglycerides (mg/dL), median (range) & $162.3(49.0-675.0)$ & $169.0(48.0-680.0)$ \\
\hline Apolipoprotein A-I (mg/dL), mean (SD) & $145.8(21.62)$ & $146.6(23.99)$ \\
\hline Apolipoprotein B (mg/dL), mean (SD) & $114.0(25.99)$ & $113.7(28.06)$ \\
\hline
\end{tabular}


colesevelam and placebo groups $(-0.58$ and $-0.70 \mathrm{~kg}$, respectively).

\section{Efficacy}

From baseline to Week 24, treatment with colesevelam, compared with placebo, resulted in significant least-squares (LS) mean reductions in A1C (treatment difference: $-0.3 \%$; $p=0.01$; primary efficacy variable), LDL cholesterol $(-13.6 \mathrm{mg} / \mathrm{dL} ; p<0.0001)$, total cholesterol $(-9.8 \mathrm{mg} / \mathrm{dL}$; $p=0.0017)$, non-HDL cholesterol $(-11.1 \mathrm{mg} / \mathrm{dL} ; p=0.0004)$, and apoB levels $(-7.0 \mathrm{mg} / \mathrm{dL} ; p=0.0002)$. A significant median increase in triglycerides (treatment difference: $16.5 \mathrm{mg} / \mathrm{dL}$; $p<0.05$ ) and LS mean increase in apoA-I levels (treatment difference: $3.4 \mathrm{mg} / \mathrm{dL} ; p<0.05$ ) was also observed with colesevelam compared with placebo [22]. The percent changes in lipid parameters from baseline to Week 24 are shown in Table 2. Colesevelam resulted in statistically significant mean percent reductions in total cholesterol, LDL-C, non-HDL-C, apoB, and apoA-I levels, and a statistically significant median percent increase in triglycerides, compared with placebo.

\section{Nuclear Magnetic Resonance Findings}

Subjects who received colesevelam, compared with placebo, had significant reductions in total LDL-P (baseline: $1,611 \mathrm{nmol} / \mathrm{L}$; LS mean treatment difference: $-143 \mathrm{nmol} / \mathrm{L} ; p<0.0001$ ), as well as large (baseline: $339 \mathrm{nmol} / \mathrm{L} ;-60 \mathrm{nmol} / \mathrm{L} ; p=0.002$ ), small (baseline: $1,203 \mathrm{nmol} / \mathrm{L} ;-82 \mathrm{nmol} / \mathrm{L} ; p<0.05)$, and very small LDL-P (baseline: $963 \mathrm{nmol} / \mathrm{L} ;-73 \mathrm{nmol} / \mathrm{L} ; p=0.03$; Table 3). Although the subjects who received colesevelam had both improvements in glycemic control and reductions in LDL-P, post-hoc correlation analysis showed no significant correlations between changes in LDL-P and changes in A1C or FPG. Change in LDL particle size was not significantly different between the treatment groups (Fig. 1).

Subjects in the colesevelam group, compared with the placebo group, had a nonsignificant reduction in total VLDL and chylomicron particle concentration (LS mean treatment difference: $-1 \mathrm{nmol} / \mathrm{L} ; p=0.82$ ) resulting from a significant reduction in small VLDL-P $(-5 \mathrm{nmol} / \mathrm{L} ; p=0.03$; Table 3$)$. An increase in VLDL particle size was also seen with colesevelam ( $p=0.001$; Fig. 1$)$.

Treatment with colesevelam relative to placebo was also associated with a nonsignificant increase in total HDL-P $(0.6 \mu \mathrm{mol} / \mathrm{L} ; p=0.20)$ attributable to significant increases in large $(0.5 \mu \mathrm{mol} / \mathrm{L} ; p=0.007)$ and medium $(0.8 \mu \mathrm{mol} / \mathrm{L}$; $p=0.02$ ) HDL-P (Fig. 1). HDL particle size was also increased relative to placebo in subjects treated with colesevelam ( $p<0.0001$; Fig. 1).

Subjects who received colesevelam, compared with placebo, also had statistically significant percent changes from
Table 2 Percent change in lipid parameters

\begin{tabular}{|c|c|c|c|c|}
\hline \multirow[t]{3}{*}{ Parameter } & \multirow[t]{3}{*}{$N$} & \multicolumn{3}{|c|}{ Percent change from baseline } \\
\hline & & \multirow[b]{2}{*}{ LS mean } & \multicolumn{2}{|c|}{ Treatment difference } \\
\hline & & & LS mean & $p$-value \\
\hline \multicolumn{5}{|c|}{ Total cholesterol, mg/dL } \\
\hline Placebo & 160 & 2 & -5 & $<0.001$ \\
\hline Colesevelam & 164 & -3 & & \\
\hline \multicolumn{5}{|l|}{ LDL-C, mg/dL } \\
\hline Placebo & 160 & 1 & -11 & $<0.0001$ \\
\hline Colesevelam & 162 & -10 & & \\
\hline \multicolumn{5}{|l|}{ HDL-C, mg/dL } \\
\hline Placebo & 160 & -0.1 & 2 & 0.31 \\
\hline Colesevelam & 164 & 2 & & \\
\hline \multicolumn{5}{|c|}{ Non-HDL-C, mg/dL } \\
\hline Placebo & 160 & 3 & -7 & $<0.001$ \\
\hline Colesevelam & 164 & -4 & & \\
\hline \multicolumn{5}{|c|}{ Triglyceride, $\mathrm{mg} / \mathrm{dL}^{\mathrm{a}}$} \\
\hline Placebo & 160 & 6 & 10 & 0.03 \\
\hline Colesevelam & 164 & 15 & & \\
\hline \multicolumn{5}{|l|}{ apoA-I, mg/dL } \\
\hline Placebo & 160 & 0.9 & 2 & 0.04 \\
\hline Colesevelam & 164 & 3 & & \\
\hline \multicolumn{5}{|l|}{ apoB, mg/dL } \\
\hline Placebo & 160 & 0.9 & -6 & $<0.001$ \\
\hline Colesevelam & 164 & -6 & & \\
\hline
\end{tabular}

Reported changes are measured from baseline to Week 24 with last observation carried forward (intent-to-treat population)

apoA-I apolipoprotein A-I, apo B apolipoprotein $\mathrm{B}, H D L-C$ high-density lipoprotein cholesterol, $L D L-C$ low-density lipoprotein cholesterol, $L S$ least squares

${ }^{\text {a }}$ Parameter is not normally distributed. The median value is reported rather than the mean value

baseline in total LDL-P (LS mean percent treatment difference: $-10 \% ; p<0.0001)$, small VLDL-P $(-13 \% ; p<0.05)$, and medium HDL-P $(167 \% ; p=0.002)$. The percent changes were not significantly different between colesevelam and placebo for the other particle classes.

\section{Safety}

Overall, $16(9.4 \%)$ and $33(18.9 \%)$ subjects in the placebo and colesevelam groups, respectively, had an adverse event (AE) considered related to study medication. Hypoglycemia occurred at a higher incidence in the colesevelam treatment group compared with placebo (4.0\% vs. $0.6 \%)$ and was generally mild overall. The majority of AEs were mild or moderate in severity. As reported in the primary study publication [22], a serious $\mathrm{AE}$ was reported by $4.0 \%$ and $1.8 \%$ of subjects in the colesevelam and placebo groups, respectively, 
Table 3 Change in lipid particle concentrations

\begin{tabular}{|c|c|c|c|c|}
\hline \multirow[t]{3}{*}{ Parameter } & \multirow[t]{3}{*}{ Baseline } & \multirow[t]{3}{*}{ Endpoint } & \multicolumn{2}{|c|}{ Change from baseline } \\
\hline & & & & $\begin{array}{l}\text { Treatment } \\
\text { difference }\end{array}$ \\
\hline & & & LS mean & LS mean $p$-value \\
\hline
\end{tabular}

Total LDL-P, nmol/L

$\begin{array}{lrrrrr}\text { Placebo } & 1,563 & 1,591 & 24 & -143 & <0.0001 \\ \text { Colesevelam } & 1,611 & 1,487 & -119 & & \end{array}$

Large LDL-P, nmol/L

$\begin{array}{lrrrrr}\text { Placebo } & 325 & 328 & 2 & -60 & 0.002 \\ \text { Colesevelam } & 339 & 278 & -59 & & \\ \text { Medium small LDL-P, nmol/L } & & & \\ \begin{array}{l}\text { Placebo } \\ \text { Colesevelam }\end{array} & 236 & 240 & 3 & -9 & 0.31 \\ \text { 240 } & 234 & -6 & & \end{array}$

Small LDL-P, nmol/L

$\begin{array}{lllrrr}\text { Placebo } & 1,169 & 1,195 & 22 & -82 & <0.05 \\ \text { Colesevelam } & 1,203 & 1,140 & -60 & & \end{array}$

Very small LDL-P, nmol/L

$\begin{array}{llll}\text { Placebo } & 933 & 955 \\ \text { Colesevelam } & 963 & 906 & -54\end{array}$

Total VLDL-P/chylomicron, nmol/L

\begin{tabular}{|c|c|c|c|c|c|}
\hline Placebo & 86 & 92 & 6 & -0.8 & 0.82 \\
\hline Colesevelam & 88 & 94 & 5 & & \\
\hline \multicolumn{6}{|c|}{ arge VLDL/chylomicron, $\mathrm{nmol} / \mathrm{L}$} \\
\hline Placebo & 6 & 7 & 0.7 & 1 & 0.08 \\
\hline Colesevelam & 6 & 8 & 2 & & \\
\hline \multicolumn{6}{|c|}{ Medium VLDL, nmol/L } \\
\hline Placebo & 38 & 41 & 4 & 4 & 0.17 \\
\hline Colesevelam & 38 & 45 & 7 & & \\
\hline \multicolumn{6}{|c|}{ mall VLDL, nmol/L } \\
\hline Placebo & 42 & 44 & 2 & -5 & 0.03 \\
\hline Colesevelam & 45 & 41 & -3 & & \\
\hline \multicolumn{6}{|c|}{ otal HDL-P, umol/L } \\
\hline Placebo & 31 & 32 & 0.4 & 0.6 & 0.20 \\
\hline Colesevelam & 31 & 32 & 1 & & \\
\hline \multicolumn{6}{|c|}{ arge HDL-P, umol/L } \\
\hline Placebo & 5 & 5 & -0.2 & 0.5 & 0.007 \\
\hline Colesevelam & 5 & 5 & 4 & & \\
\hline \multicolumn{6}{|c|}{ Medium HDL-P, umol/L } \\
\hline Placebo & 3 & 3 & 0.2 & 0.8 & 0.02 \\
\hline Colesevelam & 3 & 4 & 1 & & \\
\hline \multicolumn{6}{|c|}{ mall HDL-P, umol/L } \\
\hline Placebo & 24 & 24 & 0.3 & -0.8 & 0.10 \\
\hline Colesevelam & 24 & 23 & -0.5 & & \\
\hline
\end{tabular}

Reported changes are measured from baseline to Week 24 with last observation carried forward (intent-to-treat population)

$H D L-P$ high-density lipoprotein particle concentration, $L D L-P$ low-density lipoprotein particle concentration, $L S$ least squares, $V L D L-P$ very low-density lipoprotein particle concentration

${ }^{\mathrm{a}} p$-values are not adjusted for multiple comparisons and none were considered by investigators to be related to study medication. The most frequently reported AEs were back pain $(5.1 \%)$ and headache $(4.6 \%)$ in the colesevelam group and urinary tract infection $(8.8 \%)$ and nasopharyngitis $(5.3 \%)$ in the placebo group. There were no clinically important differences observed between the colesevelam and placebo groups in safety laboratory parameters.

\section{Discussion}

This study in adults with T2DM of relatively short duration (mean 4.1 years overall) demonstrated generally favorable changes to the lipoprotein particle profile among those who received colesevelam as an antidiabetes monotherapy, compared with those who received placebo. Significant effects were seen on LDL particles (a reduction in total LDL-P largely attributable to reductions in small and very small LDL-P, as well as large LDL-P), VLDL particles (an increase in VLDL particle size with an accompanying reduction in small VLDL-P), and HDL particles (an increase in HDL particle size with accompanying increases in large and medium HDL-P).

It is increasingly recognized that the lipoprotein profile affects CVD risk, although the influence of lipoprotein size as an independent predictor of CVD risk is less clear than that of lipoprotein concentration [5, 11, 25-27]. LDL-P is predictive for coronary endothelial dysfunction and CVD risk $[5,6]$. In one study, LDL-P was the strongest predictor of future CVD events of the lipoprotein parameters determined by NMR, with the risk for subsequent CVD events among subjects in the highest quartile of LDL-P being $>4$-fold that of the risk among subjects in the lowest quartile [5]. LDL-P remained significant even after adjusting for other lipid parameters, suggesting that LDL-P provides additional risk data beyond that provided by traditional lipoprotein parameters. Increased HDL-P and large HDL have also been shown to be associated with a reduction in CVD [6, 10, 25].

Importantly, LDL-P may be a better predictor of CVD than LDL cholesterol [4, 7-9]. Many patients with increased cardiometabolic risk have relatively normal LDL cholesterol levels but have abnormal distributions of lipoprotein particles $[4,9]$. Thus, LDL cholesterol incompletely measures the risk for coronary artery disease (CAD) [6, 8, 9, 28]. In particular, since many patients with metabolic syndrome have a relatively normal LDL cholesterol level but an elevated LDL-P, traditional monitoring of LDL cholesterol may underestimate CVD risk [28]. Our finding that colesevelam reduced LDL cholesterol levels and LDL-P (primarily through reductions in small and very small LDL-P) reflects a shift to a less atherogenic profile. Small LDL particles have been shown in several studies to be predictive of CVD events and CAD [11, 29, 30]. 
Fig. 1 Least-squares mean change in lipid particle size, from baseline to Week 24, with last observation carried forward. $H D L$ high-density lipoprotein, $L D L$ low-density lipoprotein, $V L D L$ very low-density lipoprotein

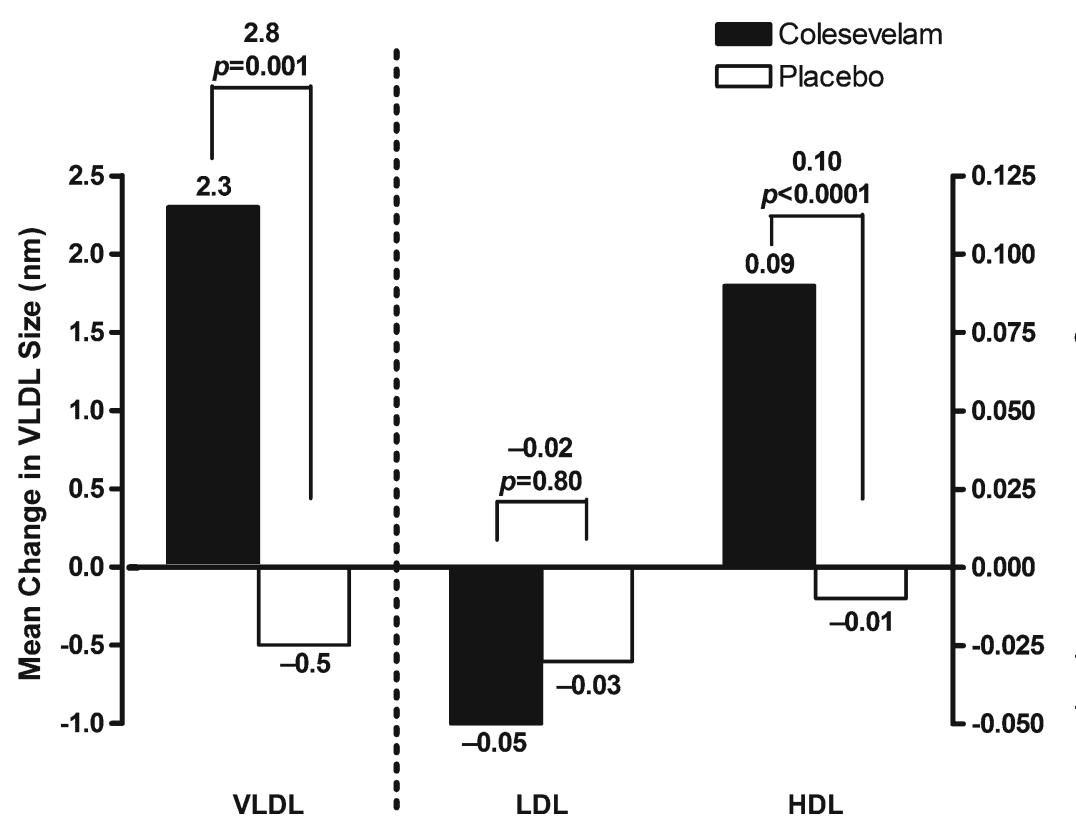

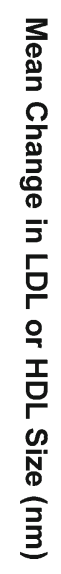

These results are in line with previous studies showing that colesevelam decreases LDL-P and increases LDL particle size in subjects with hypercholesterolemia [31], prediabetes [32], or T2DM $[20,21]$. In the previous studies in subjects with T2DM [20, 21], as in the current study, the reduction in LDL$\mathrm{P}$ was largely attributable to reductions in the smaller LDL particle subclasses: small LDL-P, as well as very small LDL-P (assessed in the current study). The reduction in very small LDL-P with colesevelam is notable given that such an effect is not seen with all other lipid-lowering agents, with some even showing an increase in this parameter [33, 34].

Despite the increase in large VLDL-P, colesevelam reduced glucose levels, which suggests that the increase in large VLDL-P does not result from worsened insulin resistance. There was also a lack of correlation between reductions in LDL-P and reductions in A1C or FPG. This may reflect the current understanding of colesevelam's independent mechanisms of LDL cholesterol lowering (via deactivation of the farnesoid $\mathrm{X}$ receptor in the intestine) and glucose lowering (via stimulation of TGR5 in the colon, secretion of the incretin hormone glucagon-like peptide1 , and reduced hepatic glucose production through suppression of glycogenolysis) [35].

A strength of this study is that the observed lipid effects can be directly attributed to colesevelam since only a small proportion of subjects administered colesevelam was on preexisting statin therapy. However, the small portion of subjects on statin therapy also limits the ability to analyze cohorts based on the use of a statin in combination with colesevelam versus colesevelam therapy alone. Consequently, it would be difficult to extrapolate the study results to determine the effect of colesevelam in patients with T2DM and elevated LDLcholesterol levels who were already taking a statin. However, it is noteworthy that a pooled analysis of data from three pivotal studies in patients with T2DM showed that adding colesevelam to concomitant statin therapy significantly reduced LDL cholesterol by an additional $16 \%$ compared with a $1 \%$ reduction in patients receiving placebo plus concomitant statin therapy $(p<0.0001)$ [36]. In addition, compared with previous studies assessing the lipoprotein particle profile, subjects in the present study were not taking other antihyperglycemic medications such as metformin and/or sulfonylurea as they had in the previous studies [20, 21]. Thus, the effect of colesevelam on the lipid profile described in the present study would not have been influenced by prior or concomitant exposure to other antidiabetes agents.

\section{Conclusions}

In subjects with T2DM, colesevelam as an antidiabetes monotherapy produced generally favorable changes in the lipoprotein particle profile compared with placebo.

Acknowledgments Bret Fulton, Alan J. Klopp, PhD, and Sushma Soni of inScience Communications, Springer Healthcare, provided medical writing support funded by Daiichi Sankyo, Inc.

Funding/Support The study was supported by Daiichi Sankyo, Inc.

Conflict of Interest Dr Rosenson has served on advisory boards for Daiichi Sankyo, Inc. and LipoScience Inc., and he has stock ownership in LipoScience Inc. Dr Rigby has received remuneration from Daiichi Sankyo, Inc. for participation in clinical trials, and has served on an advisory board for Daiichi Sankyo, Inc. Dr Jones was employed by Daiichi Sankyo, Inc. at the time of the study. Dr Chou is employed by Daiichi Sankyo Pharma Development. 
Open Access This article is distributed under the terms of the Creative Commons Attribution License which permits any use, distribution, and reproduction in any medium, provided the original author(s) and the source are credited.

\section{References}

1. Krauss RM. Lipids and lipoproteins in patients with type 2 diabetes. Diabetes Care. 2004;27(6):1496-504.

2. Garvey WT, Kwon S, Zheng D, Shaughnessy S, Wallace P, Hutto A, et al. Effects of insulin resistance and type 2 diabetes on lipoprotein subclass particle size and concentration determined by nuclear magnetic resonance. Diabetes. 2003;52(2):453-62.

3. Mora S, Otvos JD, Rosenson RS, Pradhan A, Buring JE, Ridker PM. Lipoprotein particle size and concentration by nuclear magnetic resonance and incident type 2 diabetes in women. Diabetes. 2010;59(5):1153-60.

4. Brunzell JD, Davidson M, Furberg CD, Goldberg RB, Howard BV, Stein $\mathrm{JH}$, et al. Lipoprotein management in patients with cardiometabolic risk: consensus statement from the American Diabetes Association and the American College of Cardiology Foundation. Diabetes Care. 2008;31(4):811-22.

5. Blake GJ, Otvos JD, Rifai N, Ridker PM. Low-density lipoprotein particle concentration and size as determined by nuclear magnetic resonance spectroscopy as predictors of cardiovascular disease in women. Circulation. 2002;106(15):1930-7.

6. Ford MA, McConnell JP, Lavi S, Rihal CS, Prasad A, Sandhu GS, et al. Coronary artery endothelial dysfunction is positively correlated with low density lipoprotein and inversely correlated with high density lipoprotein subclass particles measured by nuclear magnetic resonance spectroscopy. Atherosclerosis. 2009;207(1):111-5.

7. Cromwell WC, Otvos JD. Heterogeneity of low-density lipoprotein particle number in patients with type 2 diabetes mellitus and lowdensity lipoprotein cholesterol < $100 \mathrm{mg} / \mathrm{dl}$. Am J Cardiol. 2006;98(12): 1599-602.

8. El Harchaoui K, van der Steeg WA, Stroes ESG, Kuivenhoven JA, Otvos JD, Wareham NJ, et al. Value of low-density lipoprotein particle number and size as predictors of coronary artery disease in apparently healthy men and women: the EPIC-Norfolk Prospective Population Study. J Am Coll Cardiol. 2007;49(5):547-53.

9. Otvos JD, Mora S, Shalaurova I, Greenland P, Mackey RH, Goff Jr DC. Clinical implications of discordance between low-density lipoprotein cholesterol and particle number. J Clin Lipidol. 2011;5(2): $105-13$.

10. Mackey RH, Greenland P, Goff Jr DC, Lloyd-Jones D, Sibley CT, Mora S. High-density lipoprotein cholesterol and particle concentrations, carotid atherosclerosis, and coronary events: MESA (Multi-Ethnic Study of Atherosclerosis). J Am Coll Cardiol. 2012;60(6):508-16.

11. Otvos JD, Collins D, Freedman DS, Shalaurova I, Schaefer EJ, McNamara JR, et al. Low-density lipoprotein and highdensity lipoprotein particle subclasses predict coronary events and are favorably changed by gemfibrozil therapy in the Veterans Affairs High-Density Lipoprotein Intervention Trial. Circulation. 2006; 113(12):1556-63.

12. Parish S, Offer A, Clarke R, Hopewell JC, Hill MR, Otvos JD, et al. Lipids and lipoproteins and risk of different vascular events in the MRC/BHF Heart Protection Study. Circulation. 2012;125(20):246978 .

13. Rosenson RS, Davidson MH, Pourfarzib R. Underappreciated opportunities for low-density lipoprotein management in patients with cardiometabolic residual risk. Atherosclerosis. 2010;213(1):1-7.

14. Mikhailidis DP, Elisaf M, Rizzo M, Berneis K, Griffin B, Zambon A, et al. "European panel on low density lipoprotein (LDL) subclasses": a statement on the pathophysiology, atherogenicity and clinical significance of LDL subclasses. Curr Vasc Pharmacol. 2011;9(5):533-71.

15. Mora S, Glynn RJ, Ridker PM. High-density lipoprotein cholesterol, size, particle number, and residual vascular risk after potent statin therapy. Circulation. 2013;128(11):1189-97.

16. Bays HE, Goldberg RB, Truitt KE, Jones MR. Colesevelam hydrochloride therapy in patients with type 2 diabetes mellitus treated with metformin: glucose and lipid effects. Arch Intern Med. 2008;168(18): 1975-83.

17. Fonseca VA, Rosenstock J, Wang AC, Truitt KE, Jones MR. Colesevelam $\mathrm{HCl}$ improves glycemic control and reduces LDL cholesterol in patients with inadequately controlled type 2 diabetes on sulfonylurea-based therapy. Diabetes Care. 2008;31(8):1479-84.

18. Goldberg RB, Fonseca VA, Truitt KE, Jones MR. Efficacy and safety of colesevelam in patients with type 2 diabetes mellitus and inadequate glycemic control receiving insulin-based therapy. Arch Intern Med. 2008;168(14):1531-40.

19. Rosenstock J, Fonseca VA, Garvey WT, Goldberg RB, Handelsman Y, Abby SL, et al. Initial combination therapy with metformin and colesevelam for achievement of glycemic and lipid goals in early type 2 diabetes. Endocr Pract. 2010;16(4):629-40.

20. Goldberg RB, Rosenson RS, Hernandez-Triana E, Misir S, Jones MR. Initial combination therapy with metformin plus colesevelam improves lipoprotein particles in patients with early type 2 diabetes mellitus. J Clin Lipidol. 2012;6(4):318-24.

21. Rosenson RS, Abby SL, Jones MR. Colesevelam $\mathrm{HCl}$ effects on atherogenic lipoprotein subclasses in subjects with type 2 diabetes. Atherosclerosis. 2009;204(2):342-4.

22. Rosenstock J, Rigby SP, Ford DM, Tao B, Chou H. The glucose and lipid effects of colesevelam as monotherapy in drug-naive type 2 diabetes. Horm Metab Res. 2013. doi:10.1055/s-0033-1358759.

23. Otvos JD, Jeyarajah EJ, Bennett DW, Krauss RM. Development of a proton nuclear magnetic resonance spectroscopic method for determining plasma lipoprotein concentrations and subspecies distributions from a single, rapid measurement. Clin Chem. 1992;38(9): $1632-8$.

24. Freedman DS, Otvos JD, Jeyarajah EJ, Barboriak JJ, Anderson AJ, Walker JA. Relation of lipoprotein subclasses as measured by proton nuclear magnetic resonance spectroscopy to coronary artery disease. Arterioscler Thromb Vasc Biol. 1998;18(7):1046-53.

25. Mora S, Otvos JD, Rifai N, Rosenson RS, Buring JE, Ridker PM. Lipoprotein particle profiles by nuclear magnetic resonance compared with standard lipids and apolipoproteins in predicting incident cardiovascular disease in women. Circulation. 2009;119(7):931-9.

26. Rizzo M, Pernice V, Frasheri A, Di Lorenzo G, Rini GB, Spinas GA, et al. Small, dense low-density lipoproteins (LDL) are predictors of cardio- and cerebro-vascular events in subjects with the metabolic syndrome. Clin Endocrinol. 2009;70(6):870-5.

27. Mora S, Szklo M, Otvos JD, Greenland P, Psaty BM, Goff Jr DC, et al. LDL particle subclasses, LDL particle size, and carotid atherosclerosis in the Multi-Ethnic Study of Atherosclerosis (MESA). Atherosclerosis. 2007;192(1):211-7.

28. Rosenson RS, Otvos JD, Hsia J. Effects of rosuvastatin and atorvastatin on LDL and HDL particle concentrations in patients with metabolic syndrome: a randomized, double-blind, controlled study. Diabetes Care. 2009;32(6):1087-91.

29. Rosenson RS, Otvos JD, Freedman DS. Relations of lipoprotein subclass levels and low-density lipoprotein size to progression of coronary artery disease in the Pravastatin Limitation of Atherosclerosis in the Coronary Arteries (PLAC-I) trial. Am J Cardiol. 2002;90(2):89-94.

30. Rajman I, Kendall MJ, Cramb R, Holder RL, Salih M, Gammage MD. Investigation of low density lipoprotein subfractions as a coronary risk factor in normotriglyceridaemic men. Atherosclerosis. 1996;125(2):231-42. 
31. Rosenson RS. Colesevelam $\mathrm{HCl}$ reduces LDL particle number and increases LDL size in hypercholesterolemia. Atherosclerosis. 2006;185(2):327-30.

32. Goldberg RB, Rosenson RS, Hernandez-Triana E, Misir S, Jones MR. Colesevelam improved lipoprotein particle subclasses in patients with prediabetes and primary hyperlipidaemia. Diab Vasc Dis Res. 2013;10(3):256-62.

33. Krauss RM, Wojnooski K, Orr J, Geaney JC, Pinto CA, Liu Y, et al. Changes in lipoprotein subfraction concentration and composition in healthy individuals treated with the CETP inhibitor anacetrapib. J Lipid Res. 2012;53(3):540-7.
34. Rizzo M, Berneis K. The clinical relevance of low-densitylipoproteins size modulation by statins. Cardiovasc Drugs Ther. 2006;20(3):205-17.

35. Potthoff MJ, Potts A, He T, Duarte JA, Taussig R, Mangelsdorf DJ, et al. Colesevelam suppresses hepatic glycogenolysis by TGR5mediated induction of GLP-1 action in DIO mice. Am J Physiol Gastrointest Liver Physiol. 2013;304(4):G371-80.

36. Jialal I, Abby SL, Misir S, Nagendran S. Concomitant reduction in low-density lipoprotein cholesterol and glycated hemoglobin with colesevelam hydrochloride in patients with type 2 diabetes: a pooled analysis. Metab Syndr Relat Disord. 2009;7(3):255-8. 